\title{
Locally Adaptive Complex Wavelet-Based Demosaicing for Color Filter Array Images
}

\author{
Jan Aelterman $^{a}$, Bart Goossens ${ }^{a}$, Aleksandra Pižurica ${ }^{a}$ \\ and Wilfried Philips ${ }^{a}$ \\ ${ }^{a}$ Ghent University - TELIN - IPI - IBBT, St.-Pietersnieuwstraat 41, B-9000 Gent, Belgium \\ phone: +32926495 31, fax: +329264 4295 \\ email: Jan.Aelterman@Telin.Ugent.Be
}

\begin{abstract}
A new approach for wavelet-based demosaicing of color filter array (CFA) images is presented. It is observed that conventional wavelet-based demosaicing results in demosaicing artifacts in high spatial frequency regions of the image. By proposing a framework of locally adaptive demosaicing in the wavelet domain, the presented method proposes computationally simple techniques to avoid these artifacts. In order to reduce computation time and memory requirements even more, we propose the use of the dual tree complex wavelet transform. The results show that wavelet-based demosaicing, using the proposed locally adaptive framework, is visually comparable with state-of-the-art pixel based demosaicing. This result is very promising when considering a low complexity wavelet-based demosaicing and denoising approach.
\end{abstract}

Keywords: Demosaicing, Complex Wavelets, Bayer Pattern, Wavelet Packet, Artifacts, Wavelet Denoising

\section{BACKGROUND}

In modern digital camera design it is a paramount requirement to keep the price and power consumption down. Single-chip sensor solutions, like the Bayer color filter array $(\mathrm{CFA})^{1}$ are therefore very popular. CFA's involve alternating patterns of color filters to be applied at every pixel position. Demosaicing is then required, this is the estimation of missing color signal intensities from the subsampled color planes. Many different demosaicing methods have been developed in the past. ${ }^{2-5}$ One line of research focuses on frequency-domain interpretation of the demosaicing problem. ${ }^{6-8}$ Some authors remark a similarity with the luminance and chrominance demultiplexing in NTSC/PAL television. ${ }^{8}$ They propose an analogous, frequency-domain view of the demosaicing problem for demosaicing. This point of view results in schemes using linear filters to demultiplex the luminance and chrominance signals, which results in Fourier domain demosaicing methods. Unsurprisingly, this leads to similar artifacts as in NTSC/PAL, which are sometimes called, in line with the frequency-domain modulation viewpoint, luminance and chrominance crosstalk. From the same frequency-domain point of view, a waveletbased demosaicing algorithm was derived by Hirakawa ${ }^{9}$ et al.. Utilizing a wavelet-based demosaicing approach opens up the possibility to jointly handle the demosaicing and denoising problem in the wavelet domain. This is not only elegant, but allows for a lower computational cost, when compared to state of the art techniques. A disadvantage to these frequency domain methods is that they impose restrictive assumptions on the power spectral density of the image. When these assumptions are invalid, this results in luminance and chrominance crosstalk artifacts in the image, which is detrimental to the visual quality. We present a new (complex) wavelet-based demosaicing technique, that is able to eliminate most crosstalk artifacts thanks to a locally adaptive approach. This turns out to be possible without significantly increasing complexity. The remainder of this paper is as follows: In section 2 we explain the frequency-domain interpretation of the demosaicing problem using the Bayer pattern. In sections 3-4, we present the interpretation and demosaicing method by Hirakawa ${ }^{9}$ et al., which is the basic wavelet demosaicing method. In section 5 and 6 , we explain the novelties of our method with respect to the basic demosaicing method. In section 7 , we demonstrate the result of our proposed method in comparison with the basic wavelet demosaicing method and state-of-the-art pixel-based methods. 


\section{FREQUENCY-DOMAIN INTERPRETATION}

We will focus on the Bayer CFA, shown in figure 1. It should be noted however, that the general methods outligned in what follows are not restricted to Bayer CFA's. As proposed by Alleyson ${ }^{6}$ et al. , we view the demosaicing problem in terms of luminance and chrominance. As proposed by Hirakawa ${ }^{9}$ et al., we approximate luminance as the green signal and chrominances as the red and blue differences with this green signal. Later on, the well-known low bandwidth property of the chrominance, with respect to the luminance, will be exploited. The validity of the approximation, as well as the low pass claims can be disputed, e.g. in image regions that contain high frequency colored edges. However, the resulting crosstalk will be greatly reduced by the proposed locally adaptive wavelet approach, presented in section 6 .

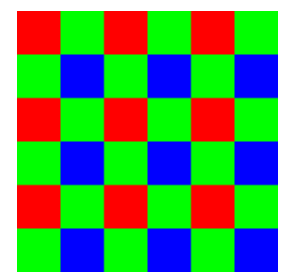

Figure 1. The Bayer Color Filter Array.

Let us define $\mathbf{n}=\left[n_{0}, n_{1}\right]$ as the position in the image and $\mathbf{x}(\mathbf{n})=[\mathrm{r}(\mathbf{n}), \mathrm{g}(\mathbf{n}), \mathrm{b}(\mathbf{n})]$ as the color signals at location $\mathbf{n}$ as would be obtained by an ideal camera with three color sensors per pixel. Also, we use the functions $P_{1}(\mathbf{n})$ and $P_{2}(\mathbf{n})$ to define the mosaic, $P_{1}(\mathbf{n})$ and $P_{2}(\mathbf{n})$ are 1 if the Bayer pattern has a red, respectively blue, value in the location $\mathbf{n}$, and 0 otherwise. The luminance-chrominance viewpoint then suggests the following interpretation of the CFA image:

$$
y(\mathbf{n})=g(\mathbf{n})+\alpha_{s, 1}(\mathbf{n})+\alpha_{s, 2}(\mathbf{n})
$$

where $\alpha_{s, 1}(\mathbf{n})$ and $\alpha_{s, 2}(\mathbf{n})$ are approximated chrominance signals and the subscript $s$ denotes subsampling:

$$
\begin{aligned}
& \alpha_{s, 1}(\mathbf{n})=\left\{\begin{array}{cc}
\alpha_{1}(\mathbf{n})=r(\mathbf{n})-g(\mathbf{n}) & \text { if } P_{1}(\mathbf{n})=1 \\
0 & \text { otherwise }
\end{array}\right. \\
& \alpha_{s, 2}(\mathbf{n})=\left\{\begin{array}{cc}
\alpha_{2}(\mathbf{n})=b(\mathbf{n})-g(\mathbf{n}) & \text { if } P_{2}(\mathbf{n})=1 \\
0 & \text { otherwise }
\end{array}\right.
\end{aligned}
$$

Following linearity of the Fourier transform, the spectrum of the mosaic image $Y(\boldsymbol{\omega})$ is equal to superposition of the spectra of its component signals $G(\boldsymbol{\omega}), A_{s, 1}(\boldsymbol{\omega})$ and $A_{s, 2}(\boldsymbol{\omega})$, with $\boldsymbol{\omega}=\left[\boldsymbol{\omega}_{0}, \boldsymbol{\omega}_{1}\right]$ the angular frequencies. The effect of the subsampling in the Fourier domain results in the introduction of aliasing copies of the original chrominance spectra (4). Aliases of $\alpha_{1}(\mathbf{n})$ and $\alpha_{2}(\mathbf{n})$ show up at frequencies $\boldsymbol{\omega} \in\{(0, \pi) ;(\pi, 0) ;(\pi, \pi)\}$ in the power spectrum of the mosaic, when compared to the power spectrum of the original signal.

$$
Y(\boldsymbol{\omega})=G(\boldsymbol{\omega})+\frac{1}{4} \sum_{k=1}^{2} \sum_{l_{0}, l_{1}=0}^{1}(-1)^{\rho_{k, l 0, l 1}} A_{k}\left(\omega_{0}+l_{0} \pi, \omega_{1}+l_{1} \pi\right)
$$

The offset of different color component patterns in the CFA translates to phase shifts $\pi \rho_{k, l_{0}, l_{1}}$ of the chrominance aliases in the spectrum, with:

$$
\rho_{k, l_{0}, l_{1}}=l_{0} P_{k}(1,0)+l_{1} P_{k}(0,1)+\left(l_{0}+l_{1}\right) P_{k}(1,1)
$$

Under the assumption that luminance and chrominance signals are sufficiently bandlimited, it can be seen that the chrominance aliases and luminance signal occupy different bands in the spectrum. They can then be demultiplexed using linear filters. The phase shifts $\pi \rho_{k, l_{0}, l_{1}}$ are different for the different chromacity signals and different aliases, which can be exploited to demultiplex the two chrominance signals $A_{1}(\omega)$ and $A_{2}(\omega)$ by solving a linear system of equations. This demultiplexing of luminance and both chrominance signals constitutes a Fourier-domain demosaicing method (e.g. ${ }^{6-8}$ ). 


\section{WAVELET DOMAIN INTERPRETATION}

Linear filtering can also be done using wavelet filter banks. This makes wavelets very suitable to perform demosaicing using the principles outligned in section 2. There are a number of potential advantages to the wavelet approach: wavelet filter banks can be constructed using separable filters, the wavelet domain is better suited for image denoising, wavelet coefficients have a spatial interpretation as well, making it possible to handle demosaicing artifacts where they occur. We will now explain the basic wavelet-based demosaicing algorithm as implemented by Hirakawa ${ }^{9}$ et al.: Let us focus on a 1 dimensional example. Suppose we have a subsampled 1-D signal $A_{s, 1}(\omega)$ and we do a 1 level discrete wavelet decomposition, which consists of a linear wavelet filters $H_{L}(\omega)$ and $H_{H}(\omega)$, followed by subsampling. The resulting wavelet band spectra, expressed in terms of the fully sampled 1-D signal $A_{1}(\omega)$ are then:

$$
A_{d, s, 1}(\omega)=\frac{1}{4}\left(H_{d}\left(\frac{\omega}{2}\right)\left(A_{1}\left(\frac{\omega}{2}\right)+A_{1}\left(\frac{\omega}{2}+\pi\right)\right)+H_{d}\left(\frac{\omega}{2}+\pi\right)\left(A_{1}\left(\frac{\omega}{2}\right)+A_{1}\left(\frac{\omega}{2}+\pi\right)\right)\right)
$$

With $d \in\{L, H\}$. If the signal $A_{s, 1}(\omega)$ had not been subsampled, the wavelet band spectra would be:

$$
A_{d, 1}(\omega)=\frac{1}{2}\left(H_{d}\left(\frac{\omega}{2}\right) A_{1}\left(\frac{\omega}{2}\right)+H_{d}\left(\frac{\omega}{2}+\pi\right) A_{1}\left(\frac{\omega}{2}+\pi\right)\right)
$$

These terms also occur in (6), apart from a scaling factor. The remaining terms are:

$$
A_{d, s, 1}(\omega)-A_{d, 1}(\omega)=\frac{1}{4}\left(H_{d}\left(\frac{\omega}{2}\right) A_{1}\left(\frac{\omega}{2}+\pi\right)+H_{d}\left(\frac{\omega}{2}+\pi\right) A_{1}\left(\frac{\omega}{2}\right)\right)
$$

Many wavelet filters have the Smith-Barnwell property:

$$
H_{d}\left(\frac{\omega}{2}+\pi\right)=c_{d} H_{d^{-1}}^{*}\left(\frac{\omega}{2}\right)
$$

With $c_{d}$ a linear phase shift factor. This means that, in a filter bank implementation, the high pass filter should be time reversed, appropriately shifted versions of the low pass filters with alternating signs: $h_{H}(k)=$ $(-1)^{k} h_{L}(N-1-k)$, as is the case for e.g. the Daubechies filters. Inserted in (8), we get:

$$
A_{d, s, 1}(\omega)-A_{d, 1}(\omega)=c_{d} \frac{1}{4}\left(H_{d^{-1}}^{*}\left(\frac{\omega}{2}+\pi\right) A_{1}\left(\frac{\omega}{2}+\pi\right)+H_{d^{-1}}^{*}\left(\frac{\omega}{2}\right) A_{1}\left(\frac{\omega}{2}\right)\right)
$$

Apart from the linear phase shift $c_{d}$, this comes down to wavelet filtering the signal with the time reversed and band inverted filter, high pass becomes low pass and vice versa. This means the wavelet subband for a subsampled signal can be seen as the sum of the wavelet subband of the original signal and the wavelet subband of the original signal filtered with the time and band inverted filter:

$$
A_{d, s, 1}(\omega)=A_{d, 1}(\omega)+A_{d^{-1, *}, 1}(\omega)
$$

Where we introduced the notation $d^{-1, *}$ for wavelet filtering with the time and band inverted filter. This additional term $A_{d^{-1, *}}^{1}(\omega)$ is the wavelet domain analogue of the aliasing copies introduced by subsampling from a Fourier domain perspective.

For a subsampled 2D image, this becomes:

$$
A_{d d, s, k}(\boldsymbol{\omega})=A_{d d, k}(\boldsymbol{\omega})+(-1)^{\rho_{k, 1,0}} A_{d^{-1, *} d, k}(\boldsymbol{\omega})+(-1)^{\rho_{k, 0,1}} A_{d d^{-1, *}, k}(\boldsymbol{\omega})+(-1)^{\rho_{k, 1,1}} A_{d^{-1, *} d^{-1, *}, k}(\boldsymbol{\omega})
$$

Where we have introduced the subscript $d d$. The first $d \in\{L, H\}$ stands for the wavelet row filter and the second $d \in\{L, H\}$ stands for the wavelet column filter. Again, the additional terms are the wavelet domain analogues of aliasing copies introduced by subsampling from a Fourier viewpoint. The phase shifts $(-1)^{\rho_{k, 1,0}}$ result from the specific orientation of the subsampled grid. 


\section{WAVELET DEMOSAICING}

For the sake of clarity we will now explain the basic wavelet demosaicing method as implemented by Hirakawa ${ }^{9}$ et al. Several assumptions are made. The first assumption is that the green signal is an approximation of the luminance, and is bandlimited to $\frac{3}{4}$ of the Nyquist bandwidth. The second assumption is the red-green and blue-green differences, $\alpha_{1}(\mathbf{n})$ and $\alpha_{2}(\mathbf{n})$ (see (1)) are bandlimited to $\frac{1}{4}$ of the Nyquist bandwidth. As mentioned in section 2, the validity of this approximation can be disputed. One of the novelties of this papier lies in the detection and solution of problems associated with this approximation. In general, the approximation is quite good, as shown in figure 2. A color photograph was chosen and the normalized radially averaged power spectrum is shown for both the difference signal $\alpha_{1}$ (n) (i.e. the red - green signal), shown as the dotted line and the green signal, shown as the full line. It's clear that the red-green signal has lower bandwidth than the green signal. Under these assumptions, the mosaic spectrum $(Y(\boldsymbol{\omega})$ from $(4))$ can be schematically interpreted as in figure

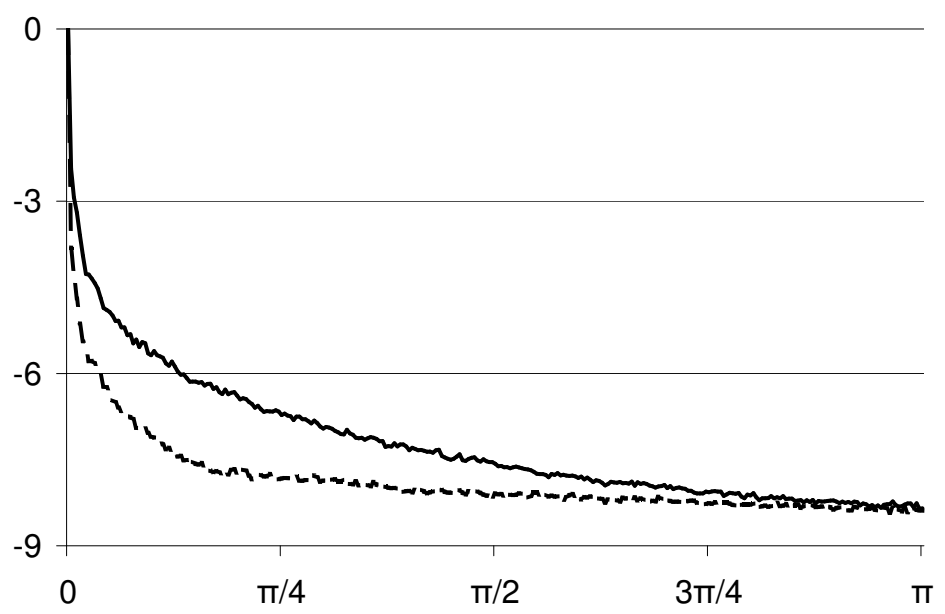

Figure 2. Normalized Radially averaged power spectrum for a color photograph. The dotted line represents the red-green difference signal, the full line represents the green signal.

3. The spectra $A_{s, 1}(\boldsymbol{\omega})$ and $A_{s, 2}(\boldsymbol{\omega})$ overlap, but have a relative phase shift of either 0 or $\pi$, depending on the band in the spectrum. This property was proven in section 2 and will enable us to demultiplex $A_{s, 1}(\boldsymbol{\omega})$ and $A_{s, 2}(\boldsymbol{\omega})$. It is visualized by the horizontal, respectively vertical stripes pattern.

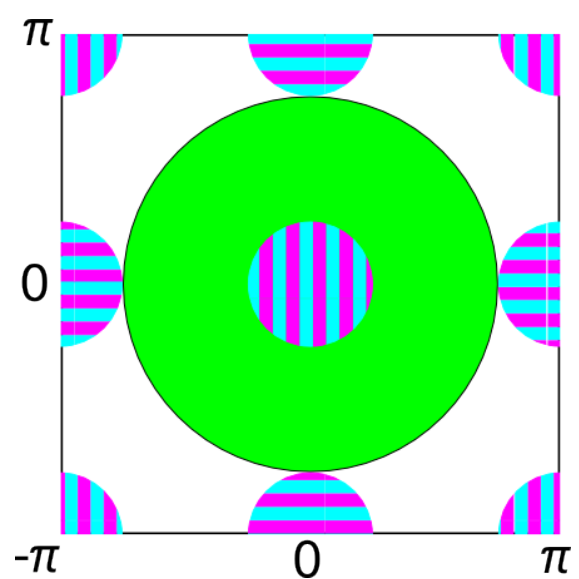

Figure 3. Schematic interpretation of the mosaic image spectrum $Y(\boldsymbol{\omega}) . G(\boldsymbol{\omega})$ is symbolized by the green disk. The horizontal, respectively vertical stripes signify different phase differences between the overlapping magenta disk, from $A_{s}^{1}(\boldsymbol{\omega})$ and the cyan disk, from $A_{s}^{2}(\boldsymbol{\omega})$. 
In order to be able to separate green from the red-green and blue-green difference signals under the assumptions, a two level wavelet packet transform suffice. This is because each band in the two level wavelet packet transform has a frequency support of approximately $1 / 4$ of the original signal bandwidth. The luminance bandwidth of $3 / 4$ can then be separated from the chrominance bandwidth of $1 / 4$. We will denote the subband of the two level wavelet packet decomposition with the double subscript dd,dd. The first dd signifies the horizontal and vertical filters used in the first level. The last dd signifies the horizontal and vertical filters used in the second level.

\subsection{Demosaicing the scaling coefficients}

Demosaicing in the wavelet domain means we have to reconstruct three wavelet coefficients, i.e. one coefficient for every colorband of the color image, from one wavelet coefficient (from the mosaic image). Given the frequency support of the different subbands in figure 3, it becomes clear that the main problem will be in demosaicing the low pass coefficients. Here, both difference signals $A_{L L, L L, 1}(\boldsymbol{\omega}), A_{L L, L L, 2}(\boldsymbol{\omega})$, as well as the green signal $G_{L L, L L}(\boldsymbol{\omega})$ are present. We will now focus on demultiplexing these three signals. The LL,LL scaling coefficients of the mosaic can be written as:

$$
Y_{L L, L L}(\boldsymbol{\omega})=A_{L L, L L, s, 1}(\boldsymbol{\omega})+A_{L L, L L, s, 2}(\boldsymbol{\omega})+G_{L L, L L}(\boldsymbol{\omega})
$$

Given the bandwidth assumptions that were made and using (12), we can express this equation in terms of the fully sampled spectra of $\alpha_{k}(\mathbf{n})$ and $g(\mathbf{n})$ and simplify:

$$
Y_{L L, L L}(\boldsymbol{\omega}) \approx A_{L L, L L, 1}(\boldsymbol{\omega})+A_{L L, L L, 2}(\boldsymbol{\omega})+G_{L L, L L}(\boldsymbol{\omega})
$$

This means the alias copies of the chrominance do not have sufficient bandwidth to extend into the lowpass band and corrupt the scaling coefficients. If we take a look at the HL,LL subband, again using the bandwidth assumptions and (12), we get:

$$
Y_{H L, L L}(\boldsymbol{\omega}) \approx(-1)^{\rho_{1,1,0}} A_{L^{*} L, L L, 1}(\boldsymbol{\omega})+(-1)^{\rho_{2,1,0}} A_{L^{*} L, L L, 2}(\boldsymbol{\omega})
$$

Where the complex conjugation of the lowpass filter results from the filter inversion explained in (11). This is an interesting result, as it means that the HL,LL subband of the mosaic contains the lowpass chrominance signals, but filtered with time inverted scaling filters. We are interested in the scaling coefficients of the chrominance signals filtered with the proper (i.e. not time inverted) scaling filters. Now let us assume we used the time inverted high pass filter in the first scale. The we get:

$$
Y_{H^{*} L, L L}(\boldsymbol{\omega}) \approx(-1)^{\rho_{1,1,0}} A_{L L, L L, 1}(\boldsymbol{\omega})+(-1)^{\rho_{2,1,0}} A_{L L, L L, 2}(\boldsymbol{\omega})
$$

Using the same trick on the HH,LL subband of the mosaic, we get:

$$
Y_{H^{*} H^{*}, L L}(\boldsymbol{\omega}) \approx(-1)^{\rho_{1,1,1}} A_{L L, L L, 1}(\boldsymbol{\omega})+(-1)^{\rho_{2,1,1}} A_{L L, L L, 2}(\boldsymbol{\omega})
$$

Using the definition in (5) and the Bayer mosaic, one can see that the vector of coefficients of $A_{L L, L L, 1}(\boldsymbol{\omega})$ and $A_{L L, L L, 2}(\boldsymbol{\omega})$ in (16) is always linearly independent from the vector of coefficients in (17). This difference in the relative phase shift between the two signals was previously mentioned and symbolized by the horizontal, respectively vertical stripes pattern in figure 3. Solving the linear system of equations (16) and (17) will always yield a unique solution, thereby demultiplexing the scaling coefficients $A_{L L, L L, 1}(\boldsymbol{\omega})$ and $A_{L L, L L, 2}(\boldsymbol{\omega})$. Then, by substracting these from (14), it becomes possible to also demultiplex the scaling coefficients $G_{L L, L L}(\boldsymbol{\omega})$ for the green image:

$$
\begin{aligned}
\hat{A}_{L L, L L, 1}(\boldsymbol{\omega}) & =\frac{(-1)^{\rho_{2,1,0}} Y_{H^{*} H^{*}, L L}(\boldsymbol{\omega})-(-1)^{\rho_{2,1,1}} Y_{H^{*} L, L L}(\boldsymbol{\omega})}{(-1)^{\rho_{1,1,1}+\rho_{2,1,0}}-(-1)^{\rho_{1,1,0}+\rho_{2,1,1}}} \\
\hat{A}_{L L, L L, 2}(\boldsymbol{\omega}) & =\frac{(-1)^{\rho_{1,1,1}} Y_{H^{*} L, L L}(\boldsymbol{\omega})-(-1)^{\rho_{1,1,0}} Y_{H^{*} H^{*}, L L}(\boldsymbol{\omega})}{(-1)^{\rho_{1,1,1}+\rho_{2,1,0}}-(-1)^{\rho_{1,1,0}+\rho_{2,1,1}}} \\
\hat{G}_{L L, L L}(\boldsymbol{\omega}) & =Y_{L L, L L}(\boldsymbol{\omega})-A_{L L, L L, 1}(\boldsymbol{\omega})-A_{L L, L L, 2}(\boldsymbol{\omega})
\end{aligned}
$$

These steps effectively demosaic the lowpass subbands. 


\subsection{Demosaicing the wavelet coefficients}

From the bandwidth assumptions, we see that the frequency support of the scaling coefficients of the difference signals, $A_{L L, L L, 1}(\boldsymbol{\omega})$ and $A_{L L, L L, 2}(\boldsymbol{\omega})$, encompasses the entire bandwidth of the difference signals. When the assumptions are correct, no further steps are needed to demosaic the difference signals. This is not true for the scaling coefficients of the green signal $G_{L L, L L}(\boldsymbol{\omega})$. However, from the bandwidth assumptions on the difference signals, we know that certain bands in the spectrum can not contain any difference signal energy, as they fall outside the bandwidth of the difference signals and their aliases (figure 4). Hence, they will only contain energy from the green signal. This leads to the following demosaicing rule for the bands indicated in figure 4 :

$$
\hat{G}_{d_{1} d_{1}, d_{2} d_{2}}(\boldsymbol{\omega})=Y_{d_{1} d_{1}, d_{2} d_{2}}(\boldsymbol{\omega}) \quad \text { if } \quad d_{2} d_{2} \neq L L
$$

If the wavelet filters would be ideal and the bandwidth assumptions correct, then the combination of (18) and (19) would constitute a perfect demosaicing method.

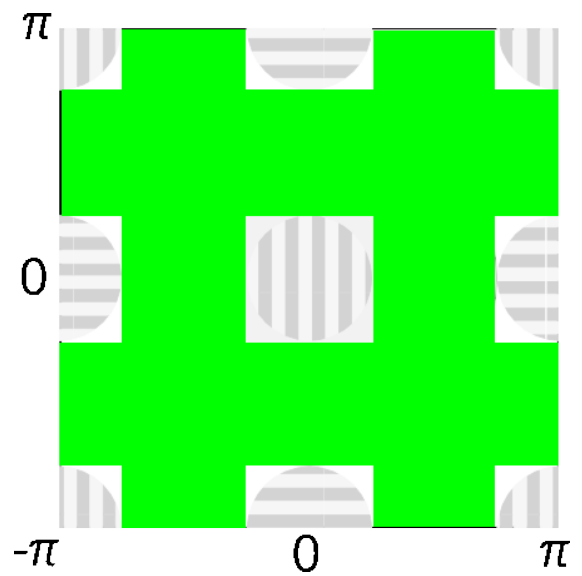

Figure 4. Highlight of the bands in the mosaic spectrum that are not assumed to contain difference signal (chromacity) or aliases.

\section{COMPLEX WAVELETS}

The dual-tree complex wavelet transform ${ }^{10,11}$ is an improvement over the more traditional discrete wavelet transform. Two properties allow for improved performance in image applications (e.g. denoising): complex wavelet processing is (nearly) shift invariant and offers far more directional selectivity. Both properties can be exploited in the wavelet demosaicing framework. It is a well known fact that although the wavelet transform is alias-free, any processing to coefficients of the decimated wavelet transform is not, which results in aliasing artifacts. ${ }^{12}$ Therefore the undecimated wavelet transform is often used (e.g. in image denoising). The undecimated wavelet transform is highly redundant. In fact, if the two level undecimated wavelet packet transform was used, there would be a redundancy factor of 16 . The two dimensional dual tree wavelet packet transform only has a redundancy factor of 4 . This is a very desirable property for a demosaicing algorithm, as it reduces computational cost as well as memory requirements. Figure 5(a) shows the ideal frequency support of the subbands of the discrete wavelet transform. The dual tree complex wavelet transform uses analytic filters. The quadrature filter in the analytic filter pair results in a signal which has a phase shift of $\pi / 2$ compared to the in-phase filter. This allows to demultiplex the positive and negative frequencies, by a linear combination of wavelet coefficients from the different filter trees. ${ }^{11}$ One can consider this as quadrant selectors in the spectrum, the four trees of the dual-tree complex wavelet transform allow to demultiplex frequency responses coming from any of the the four highlighted quadrants in the spectrum support of a DWT composition in figure 5(b).

The quadrant selector principle results in coefficients from directional, non-separable filters, but implemented using only separable, analytic wavelet filter pairs from four decimated DWT trees. Figure 6(a) shows the frequency support of one subband in the DWT decomposition. It is obvious that this is can be obtained by 


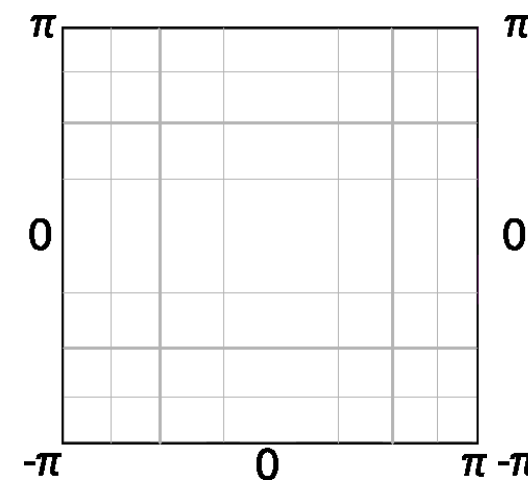

(a)

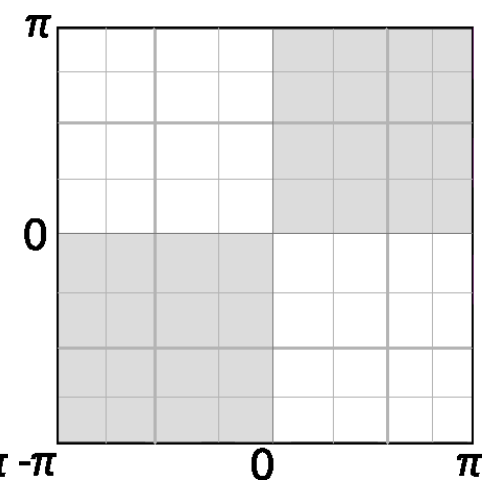

(b)

Figure 5. (a) Spectrum support of the different subbands of a 2 level discrete wavelet packet decomposition (b) Spectrum support of the different subbands of a 2 level dual-tree complex wavelet packet decomposition with the different quandrants highlighted

separable filters and also, that it lacks a clear directionality, i.e. it lacks the ability to distinguish $45^{\circ}$ from $-45^{\circ}$. The dual tree complex wavelet transform allows to demultiplex the four quadrants of the spectrum, so the support of one similar subband of the DT-CWT is shown in figure 6(b). The quadrant selection in the DT-CWT

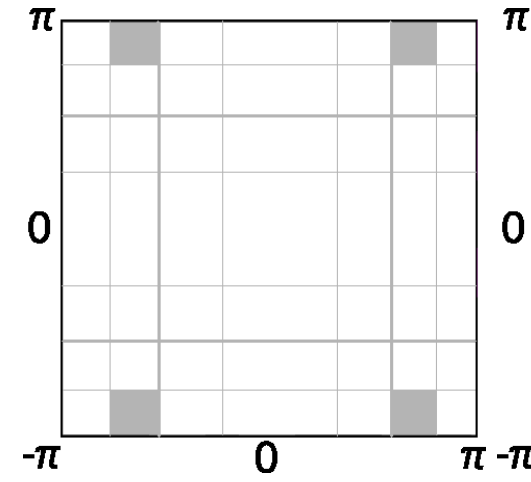

(a)

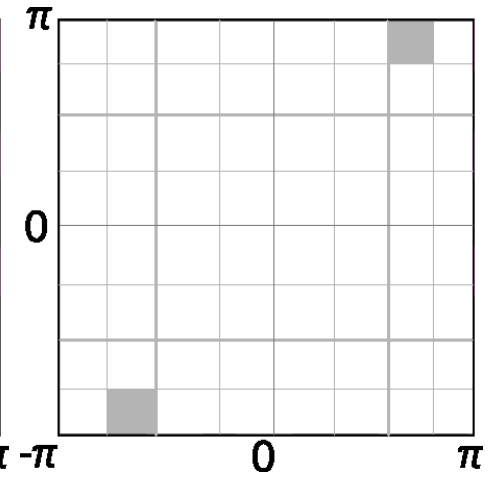

(b)

Figure 6. (a) Idealized spectrum support of one subband of the DWT (b) Idealized spectrum support of one subband of the DT-CWT

allows for a more precise selection of different bands in the spectrum. A more precise selection allows for a better isolation of problematic parts of the spectrum in order to avoid local demosaicing artifacts in a more precise, i.e. better way. This property, combined with the smaller redundancy makes the DT-CWT a very good choice for wavelet-based demosaicing.

\section{LOCALLY ADAPTIVE WAVELET DEMOSAICING}

\subsection{Color corruption artifacts}

The basic wavelet-based demosaicing algorithm outligned in section 4 suffers from disturbing demosaicing artifacts (figure 7). In this section, we will omit the suffixes denoting the real or complex part and the direction of the complex wavelets. Handling these wavelet subbands differently allows for an even greater improvement in demosaicing quality, but in order to maintain the link with DWT demosaicing and in order to simplify our notations, we will leave them out in this explanation. This way, the demosaicing method developed in this section is applicable to DT-CWT demosaicing, as well as DWT demosaicing. We will also drop the angular frequency variable, as the method outligned here operates locally adaptive, i.e. differently for different wavelet coefficients. 
Instead expressing the demosaicing equations in terms of DFT spectra, as in the previous sections, we will now apply them to the wavelet coefficients directly. We will write a wavelet coefficient as $Y_{d d, d d}$, where we drop the position index. Note that this does not change the demosaicing equations.

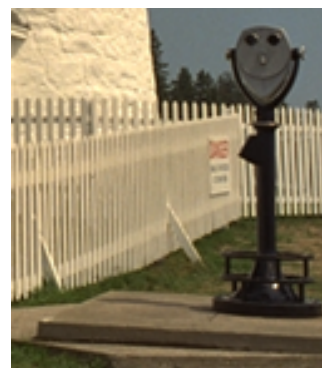

(a)

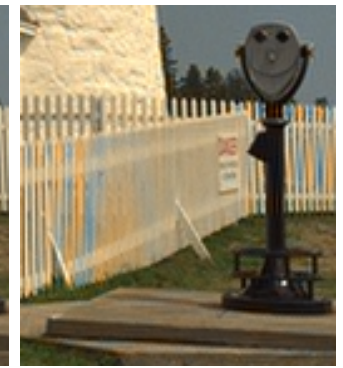

(b)

Figure 7. (a) The imaged scene (crop from the lighthouse image) (b) Result of the wavelet based demosaicing algorithm ${ }^{9}$

The color corruption problems shown in figure 7 occur when the bandwidth assumptions are not correct. Images are often viewed as consisting of smooth regions bounded by sharp edges. This is the reasoning behind the Laplacian or Gaussian scale mixture prior models used in wavelet image denoising. The same models makes it clear why the wavelet framework is preferable over the Fourier framework for demosaicing. Any sharp edges result in localized high frequencies, and will show up as large wavelet coefficients locally. The other, small wavelet coefficients are for the most part from smooth regions, where the demosaicing assumptions will be correct. This behaviour is also visible in figure 7. Demosaicing artifacts only occur in image regions with high spatial frequencies, such as the fence. High localized frequencies cause demosaicing artifacts. This is because the bandwidth assumptions made for the basic wavelet demosaicing algorithm are not correct anymore. Figure 8 illustrates this situation locally, where we have a sharp vertical edge. The green spectrum exceeds its assumed

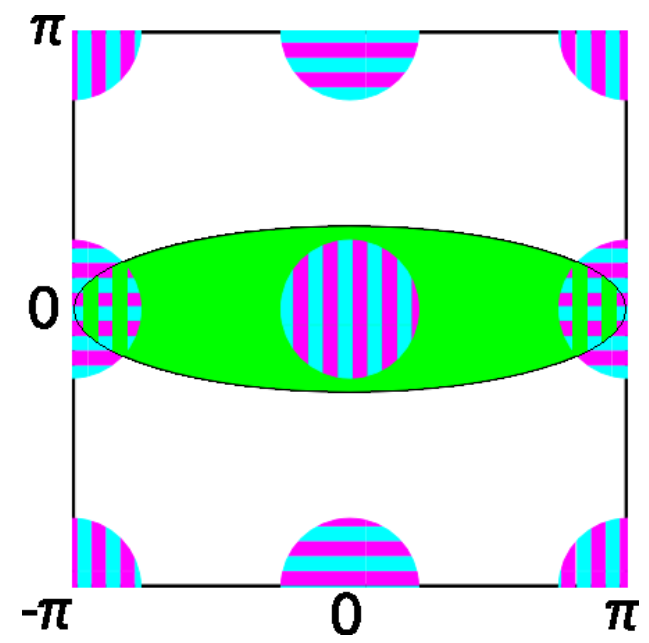

Figure 8. Schematic interpretation of the mosaic image spectrum with a sharp vertical edge. Note the overlap between the green spectrum and the chrominance spectra.

bandwidth of $3 / 4$ the total bandwidth. On figure 8 , we can see this problem very clearly, as now the bandwidth of the signal extends into the alias copies of the difference signals. In the Fourier framework we would talk of crosstalk between chrominance and luminance. In the wavelet framework, the approximation in (16) will no longer be accurate. Instead we will have:

$$
Y_{H^{*} L, L L} \approx(-1)^{\rho_{1,1,0}} A_{L L, L L, 1}+(-1)^{\rho_{2,1,0}} A_{L L, L L, 2}+G_{H^{*} L, L L}
$$


This term will make solving the linear system for $A_{L L, L L, 1}$ and $A_{L L, L L, 2}$ impossible as we now have the additional term $G_{H^{*} L, L L}$. However, it is possible to solve this problem if we assume that the bandwidth limitation is only exceeded in one direction. If we would just blindly use the reconstruction rule (18) we would get the estimate:

$$
\hat{A}_{L L, L L, 1}=A_{L L, L L, 1}-\frac{\rho_{2,1,0}}{\rho_{2,1,0} \rho_{1,1,1}-\rho_{1,1,0} \rho_{2,1,1}} G_{H^{*} L, L L}
$$

The color corruption artifacts visible in figure 7 are a result of the extra term that was introduced in (21). The low pass chrominance coefficient is locally corrupted by the addition of a term representing high frequency luminance energy. High frequency luminance energy is literally converted into low frequency chrominance energy.

\subsection{Avoiding color corruption artifacts}

Color corruption artifacts are visually very disturbing. The solution lies in exploiting redundancy in the spectrum. Again, we use the schematic interpretation of the spectrum (figure 8). So far we've been exploiting the chrominance aliases to reconstruct the lowpass chrominance signal. We can see that there are other aliases with similar phase relationship between both chrominance aliases. In the wavelet framework we see that even though $Y_{H^{*} L, L L}$ is corrupted (see equation (20)), $Y_{L H^{*}, L L}$ is not:

$$
Y_{L H^{*}, L L} \approx(-1)^{\rho_{1,1,0}} A_{L L, L L, 1}+(-1)^{\rho_{2,1,0}} A_{L L, L L, 2}
$$

So, if (22) is used instead of (20) when solving the linear system with equation (17), we get the reconstruction formulas:

$$
\begin{aligned}
\hat{A}_{L L, L L, 1} & =\frac{(-1)^{\rho_{2,1,0}} Y_{H^{*} H^{*}, L L}-(-1)^{\rho_{2,1,1}} Y_{L H^{*}, L L}}{(-1)^{\rho_{1,1,1}+\rho_{2,1,0}}-(-1)^{\rho_{1,1,0}+\rho_{2,1,1}}} \\
\hat{A}_{L L, L L, 2} & =\frac{(-1)^{\rho_{1,1,1}} Y_{L H^{*}, L L}-(-1)^{\rho_{1,1,0}} Y_{H^{*} H^{*}, L L}}{(-1)^{\rho_{1,1,1}+\rho_{2,1,0}}-(-1)^{\rho_{1,1,0}+\rho_{2,1,1}}} \\
\hat{G}_{L L, L L} & =Y_{L L, L L}-A_{L L, L L, 1}-A_{L L, L L, 2}
\end{aligned}
$$

Of course, the analog problem occurs when a horizontal edge is encountered. Then (23) will be the reconstruction rule to introduce color artifacts and (18) will be the one that avoids color corruption artifacts. It is not a simple case of choosing either (18) or (23), we have to locally adapt the demosaicing to pick the optimal set of reconstruction rules. To do that, we have to locally detect the predominant direction of the spatial frequencies in the original image. If we knew this up front, by having access to the original scene, we get the results of figure 6.2. One interpretation of this technique is as vertical or horizontal interpolation. By using the wavelet coefficient

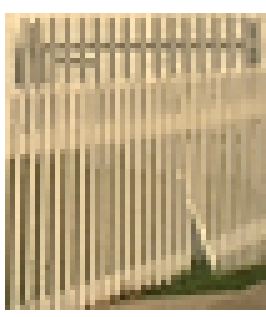

(a)

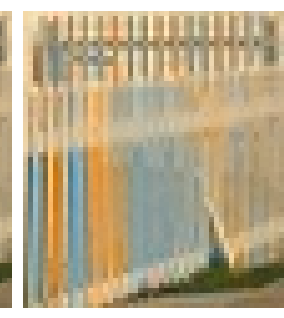

(b)

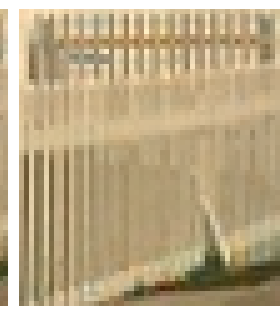

(c)

Figure 9. (a) The imaged scene (crop from the lighthouse image) (b) Demosaicing result from the wavelet based demosaicing algorithm from Hirakawa ${ }^{9}$ (c) Demosaicing result from the locally adaptive wavelet based demosaicing approach using ideal knowledge about the dominant edge direction

that is insensitive to the high signal frequency direction, we are eliminating the interference from this signal. If the signal is a vertical edge, the demosaicing uses coefficients that are insensitive to the horizontal direction. This can be interpreted as vertical interpolation. This kind of switching between horizontal and vertical edge-adaptive interpolation is a well established practice in pixel-based demosaicing (e.g. Zhang ${ }^{3}$ or Adams ${ }^{13}$ ). Our proposed 
method can hence be viewed as a wavelet domain equivalent. This is not a correct way to evaluate the locally adaptive wavelet demosaicing method, because we do not know the original image when demosaicing. Rather it is a way to validate our demosaicing rules, using ideal information about the occurrence of color corruption artifacts. An attempt to obtain this information from the mosaic is explained in section 6.3.

\subsection{Detecting color corruption artifacts}

We evaluated several methods for detecting the occurrence of color corruption artifacts locally. Again, thanks to the wavelet framework we can detect energy locally. If the corruption shown in figure 8 occurs, it will likely increase the energy of the $Y_{L H^{*}, L L}$ wavelet coefficient. Under normal circumstances, when the assumptions are correct, the equality $Y_{L H^{*}, L L}=Y_{H^{*} L, L L}$ should stand, as they only contain the same aliases of the lowpass chrominance. So, we should be able to detect problems when the equality is not correct. If the magnitude of either the wavelet coefficient $Y_{L H^{*}, L L}$ or $Y_{H^{*} L, L L}$ has been increased too much, the demosaicing rule that does not include this wavelet coefficient (either (18) or (23)) is used. This would result in demosaicing without the color corruption artifact. The result is shown in figure 10(c). The result is bad, because there are several problems with the method. The decimation involved in the DT-CWT causes aliasing in the wavelet coefficients, this leads to incorrect local estimations of the energy if the wavelet coefficient magnitude is used.

The complex wavelet framework allows to detect significant high frequencies, by using both the real and imaginary part of the complex wavelet coefficient. One of the defining properties of the CWT is that the complex wavelet coefficient magnitude is (approximately) alias-free and translation invariant * This enables detecting the energy of the high spatial frequency components, regardless of their local phase. This is yet another advantage of the DT-CWT over the decimated DWT in demosaicing. Instead of comparing $\left|Y_{L H^{*}, L L}\right|$ to $\left|Y_{H^{*} L, L L}\right|$, we will compare $Y_{R, L H^{*}, L L}^{2}+Y_{I, L H^{*}, L L}^{2}$ to $Y_{R, H^{*} L, L L}^{2}+Y_{I, H^{*} L, L L}^{2}$. The suffix $R$ signifies the real part of the complex wavelet coefficient and the $I$ signifies the imaginary part. The result of this approach is shown in figure $10(\mathrm{~d})$. The result is better, because aliasing is avoided, but it is still not perfect. One reason is the assumption that the corrupting high luminance frequencies result in an increase of the complex wavelet coefficient magnitude. It is clear from (20) that the extra term $G_{H^{*} L, L L}$ does not necessarily result in an increase of the magnitude of $Y_{H^{*} L, L L}$.

We propose an adaptation that result in more accurate estimates of the corrupted wavelet coefficients: We make use of a local spatial activity indicators (LSAI). These have been used in denoising (e.g. Pižurica ${ }^{14}$ et al.) and try to detect significant local signal energy in a more robust way by averaging neighboring wavelet coefficients in a local window. The result of using the LSAI on complex wavelet coefficient magnitude in demosaicing is shown on figure $10(\mathrm{e})$.

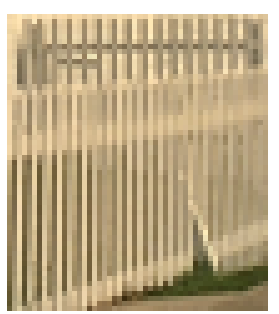

(a)

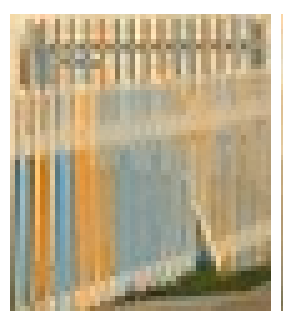

(b)

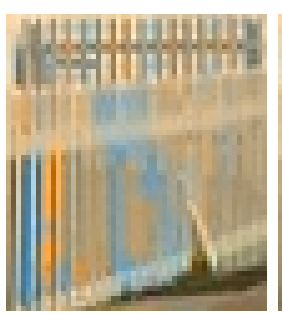

(c)

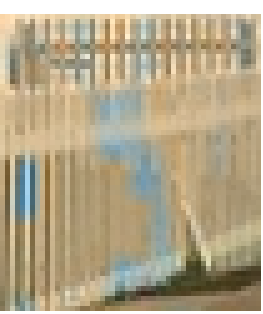

(d)

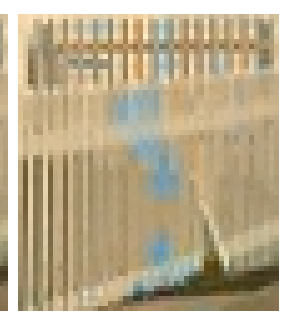

(e)

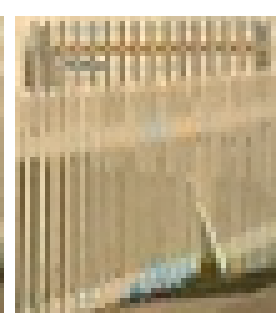

(f)

Figure 10. (a) The imaged scene (crop from the lighthouse image) (b) The basic wavelet based demosaicing algorithm. ${ }^{9}$ Different methods to detect the the occurrence of color corruption artifacts: (c) Comparing wavelet magnitudes (d) Comparing complex wavelet magnitudes (e) Comparing LSAI from complex wavelet magnitudes (f) Comparing complex wavelet magnitude products from vertically and horizontally interpolated images

A somewhat more demanding technique is to use preliminary demosaicing results, obtained using a different demosaicing technique. Combining vertically and horizontally interpolated images in order to avoid demosaicing

\footnotetext{
${ }^{*}$ Because of the requirements of compact wavelet filter supports, perfectly alias-free and translation invariant wavelet coefficients can not be obtained in practice.
} 
artifacts is a very well established practice in pixel-based demosaicing (e.g. Zhang ${ }^{3}$ et al., Adams ${ }^{13}$ et al.). Our idea is to interpolate the image using a simple directional averaging technique. We make two images: one where every missing pixel was interpolated using only pixels on the same vertical line and one where every pixel was interpolated using only pixels on the same horizontal line. Correctly interpolated edges will not cause significant high frequency energy in the direction along to the edge. In wavelet terms it means that, for the correctly interpolated edge, either $Y_{R, L H, L L}^{2}+Y_{I, L H, L L}^{2}$ or $Y_{R, L H, L L}^{2}+Y_{I, L H, L L}^{2}$ must be small. So we compare the product of these wavelet coefficient magnitudes for the horizontally interpolated image with the same product from the vertically interpolated image. The correct interpolation direction will result in a small product, so this comparison decides whether to use (18) or (23) for the wavelet coefficient in question. The demosaicing result is shown in figure $10(\mathrm{f})$.

\section{RESULTS AND CONCLUSION}

Experiments show that the proposed method greatly improves the visual quality in terms of avoiding discolorations compared to other wavelet demoaicing methods ${ }^{9}$ because of its local adaptivity. Some results are shown in figures 11(a)-11(d) and 12(a)-12(d). The locally adaptiveness of the proposed method solves color corruption artifacts in the fence of the lighthouse image and the roof tiles in the goldhill image. For most natural images, results are comparable to the state-of-the-art, pixel-based method by Zhang ${ }^{3}$ et al., while retaining the advantages of a wavelet-based demosaicing technique. These advantages include simple processing steps, demosaicing

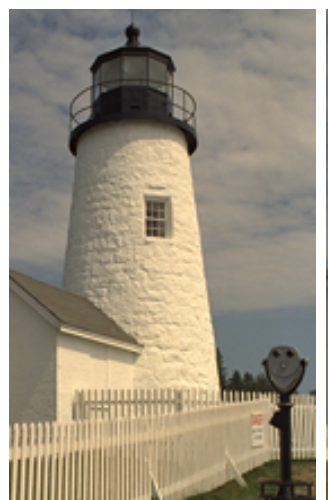

(a)

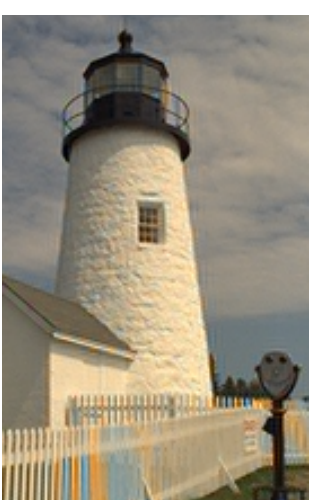

(b)

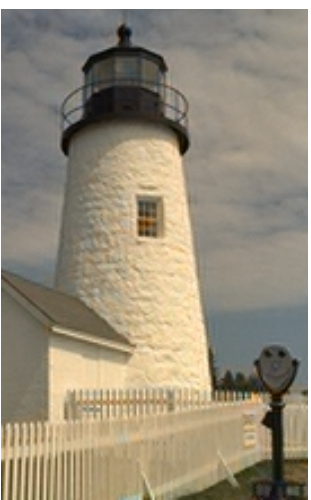

(c)

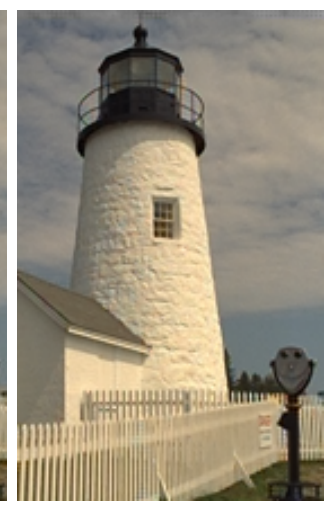

(d)

Figure 11. (a) The imaged scene (crop from the lighthouse image) (b) The basic wavelet based demosaicing algorithm ${ }^{9}$ (c) Proposed method (d) State-of-the-art pixel-based method ${ }^{3}$

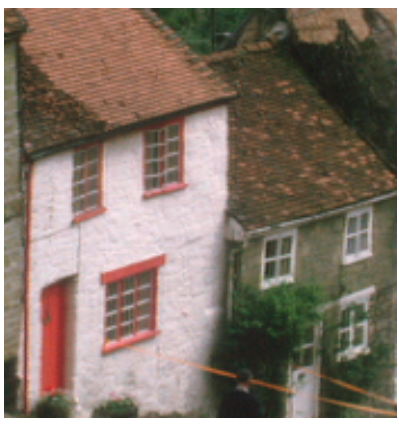

(a)

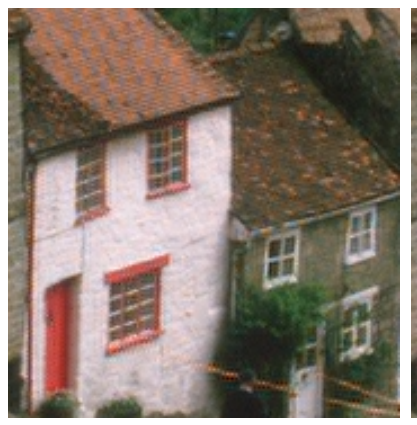

(b)

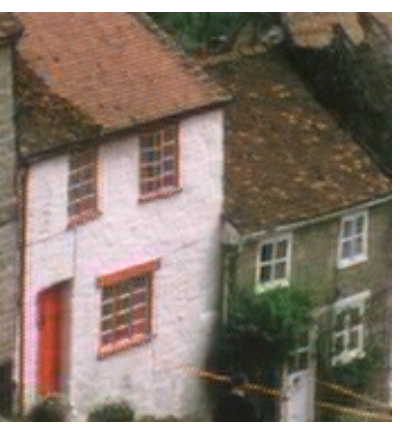

(c)

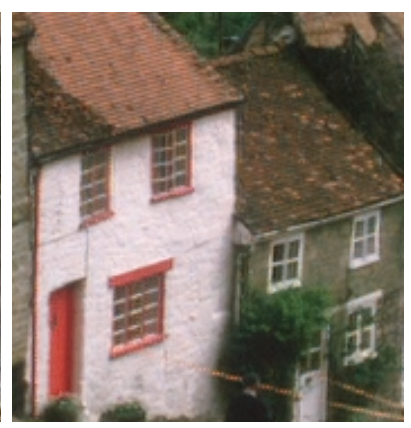

(d)

Figure 12. (a) The imaged scene (crop from the goldhill image) (b) The basic wavelet based demosaicing algorithm $^{9}$ (c) Proposed method (d) State-of-the-art pixel-based method ${ }^{3}$ 
comes down to little more than comparing coefficients and then doing a very small number of additions, if any at all. Of course there is need of a wavelet decomposition. It would be a very nice application to perform demosaicing jointly with denoising. The wavelet decomposition can then be thought of part of the denoising method and one would end up with a very simple, very fast, high quality demosaicing method.

\section{REFERENCES}

[1] E. Bayer, B., "Color imaging array," US Patent 3,971,065 (July 1976).

[2] Kimmel, R., "Demosaicing: Image reconstruction from color ccd samples," IEEE Transactions on Image Processing 8 (September 1999).

[3] Zhang, L. and $\mathrm{Wu}, \mathrm{X}$., "Color demosaicking via directional linear minimum mean square-error estimation," IEEE Transactions on Image Processing 14, 2167-2178 (December 2005).

[4] K. Gunturk, B., Altunbasak, Y., and M. Mersereau, R., "Color plane interpolation using alternating projections," IEEE Transactions on Image Processing 11, 997-1013 (September 2002).

[5] Li, X., "Demosaicing by successive approximation," IEEE Transactions on Image Processing 14, 370-379 (March 2005).

[6] Alleysson, D. and Susstrunk, S., "Linear demosaicing inspired by the human visual system," IEEE Transactions on Image Processing 14, 1-11 (April 2005).

[7] Alleysson, D. and de Lavarene, C., "Frequency selection demosacking: A review and a look ahead," in [Proc. SPIE], 6822 (2008).

[8] Dubois, E., "Frequency-domain methods for demosaicking of bayer-sampled color images," IEEE Signal Processing Letters 12, 847-850 (December 2005).

[9] Hirakawa, K., Meng, X.-L., and J. Wolfe, P., "A framework for wavelet-based analysis and processing of color filter array images with applications to denoising and demosaicing," Proc. IEEE International Conference on Acoustics, Speech, and Signal Processing, 597-600 (Apr. 2007).

[10] Kingsbury, N., "Complex wavelets for shift invariant analysis and filtering of signals," Applied and Computational Harmonic Analysis 10, 234-253 (May 2001).

[11] Selesnick, I. W., Baraniuk, R. G., and Kingsbury, N. G., "The dual-tree complex wavelet transform," IEEE Signal Processing Magazine, 123-151 (November 2005).

[12] Chen, P. and Suter, D., "Shift-invariant wavelet denoising using interscale dependency," in [IEEE Int. Conf. on Image Processing (ICIP)], (2004).

[13] Adams, J. and Hamilton, Jr., J., "Adaptive color plane interpolation in single sensor color electronic camera," US Patent 5,506,619 (1996).

[14] Pižurica, A., Philips, W., Lemahieu, I., and Acheroy, M., "A versatile wavelet domain noise filtration technique for medical imaging," IEEE Transactions on Medical Imaging 22, 323-331 (March 2003). 